\title{
Effect of a fatty meal on inflammatory markers in healthy volunteers with a family history of type 2 diabetes
}

\author{
Stephanie Madec, Valentina Corretti, Eleonora Santini, Ele Ferrannini and Anna Solini* \\ Department of Internal Medicine, University of Pisa School of Medicine, Via Roma, 67, I-56100 Pisa, Italy
}

(Received 23 August 2010 - Revised 9 December 2010-Accepted 12 January 2011 - First published online 9 March 2011)

\begin{abstract}
A family history of type 2 diabetes (T2D) confers a high risk of developing the disease, independent of that due to other common risk factors. Postprandial state is a pro-inflammatory condition associated with a transiently impaired endothelial function; an increased oxidative stress is considered as a mediator of such effects in T2D. We evaluated the short-term effect of a lipid meal on markers of early vascular damage in subjects at risk of developing T2D. A total of thirty-two healthy volunteers, divided according to the presence (FHD + ) or absence (FHD-) of a family history of T2D, underwent a fatty meal test. We measured the monocyte mRNA expressions of IL-6, IL-8 and IL-1 $\beta$, and IL-6, soluble CD40 ligand (sCD40L), vascular cellular adhesion molecule-1 (VCAM-1), intracellular adhesion molecule-1 (ICAM-1) and nitrotyrosine plasma concentrations at baseline and in the post-meal phase, relating them to the lipid profile and other biochemical parameters. The basal expression of the cytokines did not differ in FHD - and FHD + subjects; neither was it modified by the meal ingestion. IL- 6 and SCD $40 \mathrm{~L}$ plasma levels, similar in the two groups in the fasting state, did not vary after the meal. VCAM-1 and ICAM-1 increased in FHD+ subjects but not in FHD - subjects. Nitrotyrosine, similar between the FHD- and FHD+ subjects at baseline, increased more in FHD+ subjects than in FHD - subjects after the meal. In conclusion, the presence of a familial history of T2D confers an abnormal endothelial activation after an oral lipid meal, coupled with an increased oxidative stress, supporting the hypothesis of an early endothelial dysfunction already present in healthy individuals prone to develop T2D.
\end{abstract}

Key words: Type 2 diabetes: Fatty meals: Adhesion molecules: Oxidative stress

First-degree relatives of individuals with type 2 diabetes (T2D) are a good human model to test for mechanisms involved in the early development of atherosclerosis, as long before developing overt diabetes, they show insulin resistance, dyslipidaemia, increased abdominal adiposity and muscle TAG content, and reduced first-phase insulin secretion in response to glucose $\mathrm{g}^{(1,2)}$.

Postprandial hyperlipaemia may promote coronary and carotid artery disease; moreover, the magnitude of postprandial triacylglycerolaemia is related to the intima-media thickness of the carotid arteries, a surrogate marker for early atherosclerosis $^{(3,4)}$. Chronic consumption of fat-rich diets leads to atherosclerosis, inducing endothelial dysfunction and other changes in the vascular system ${ }^{(5,6)}$; these alterations are accompanied by an increased production of some inflammatory molecules. Among these, IL-1 $\beta$ promotes migration and development of smooth muscle cells within the atherosclerotic lesion and stimulates vascular endothelial growth factor synthesis, thus contributing to the development of diabetic microangiopathy ${ }^{(7)}$; IL-6 is implicated in endothelial dysfunction and vascular inflammation, and promotes insulin resistance ${ }^{(8)}$; IL-8 mediates leucocyte adhesion to endothelial cells and infiltration into the arterial wall ${ }^{(9)}$; CD40 promotes restenosis after vascular injury, and enhanced expression of the CD40 ligand in platelets and elevated plasma levels of the soluble CD40 ligand ( $\mathrm{SCD} 40 \mathrm{~L}$ ) have been reported in subjects at high risk of developing $\mathrm{CHD}^{(10-13)}$.

Circulating levels of intracellular adhesion molecule-1 (ICAM-1) and vascular cellular adhesion molecule-1 (VCAM-1) increase in diabetic patients ${ }^{(14)}$ and predict the development of the disease ${ }^{(15)}$. Evidence suggests that postprandial hyperlipaemia may stimulate the release of these molecules, with oxidative stress as the putative mediator of such effect ${ }^{(16)}$ and nitrotyrosine as a good marker of peroxynitrite and nitrosative stress generation. However, there is no information on the acute effects of a fat-rich meal on the appearance of these markers of early endothelial damage in subjects at risk of developing T2D due to a positive family history of the disease.

Abbreviations: FHD +, subjects with a family history of type 2 diabetes; FHD-, subjects without a family history of type 2 diabetes; ICAM-1, intracellular adhesion molecule-1; sCD40L, soluble CD40 ligand; T2D, type 2 diabetes; VCAM-1, vascular cellular adhesion molecule-1. 
We examined whether a lipid-rich meal modulates the synthesis of cytokines and stimulates the release of adhesion factors and parameters of oxidative stress in young healthy offspring of diabetic patients.

\section{Subjects and methods}

The participants in the study were sixteen non-smoking, healthy, young volunteers with one or both parents with T2D (FHD+) who were consecutively enrolled on a voluntry basis among post-doctoral students matching the inclusion criteria and compared with other sixteen subjects of similar age and BMI but without a family history of T2D (FHD-). Inclusion criteria were negative history of chronic illness, normal glucose tolerance (assessed by an oral glucose tolerance test) and absence of clinical or biochemical signs of ongoing inflammation (highly sensitive C-reactive protein $<0.5 \mathrm{mg} / \mathrm{l}$ ).

All subjects were asked to follow the same written diet for the 2 weeks preceding the study, a diet of $5842 \mathrm{~kJ}$ $(1400 \mathrm{kcal})$ with $16 \%$ protein, 58\% carbohydrate and $24 \%$ fat. Alcohol consumption was moderate in fourteen subjects $(<60 \mathrm{~g} /$ week $)$ and occasional in six, and four were teetotallers. None of the participants practised strenuous physical exercise or was taking any medication. The present study was conducted according to the guidelines laid down in the Declaration of Helsinki, and all procedures were approved by the Institutional Review Board of the University of Pisa (Pisa, Italy). Written informed consent was obtained from all subjects.

Participants were admitted early in the morning after a $12 \mathrm{~h}$ fast. Blood pressure was measured four times in the sitting position after a $10 \mathrm{~min}$ rest; the mean of the last two measurements was used for statistical analysis. A blood sample was taken before the test meal; then, they consumed (within $15 \mathrm{~min}$ ) a fat-enriched breakfast (butter $25 \mathrm{~g}$, bread $100 \mathrm{~g}$ and $10 \%$ fat ham $50 \mathrm{~g}$ ). The energy content of the meal was $3051 \mathrm{~kJ}(730 \mathrm{kcal})$, with $13 \%$ of energy from protein, $35 \%$ from carbohydrate and $52 \%$ from fat. At 2 and $6 \mathrm{~h}$ after the meal, blood samples were drawn for the isolation of monocytes and the determination of biochemical parameters and inflammatory molecules. Some measurements were also repeated after $12 \mathrm{~h}$.

\section{Biochemical parameters}

Plasma cholesterol and TAG concentrations were measured by standard enzymatic assays. HDL-cholesterol was measured after precipitation of apoB-containing lipoproteins, and LDL-cholesterol was calculated by the Friedewald formula. Plasma NEFA assay was performed by a spectrophotometric method (Wako Chemicals, Neuss, Germany), plasma insulin by RIA (Linco Research, Inc., St Charles, MO, USA), with an intra-assay CV of $3 \cdot 2 \%$ and an inter-assay CV of $4.0 \%$.

\section{Monocyte isolation}

Blood was collected in vacutainers containing sodium heparinate, diluted (1:1) with sterile Dulbecco's PBS, slowly laid on a warm Ficoll-Hypaque solution (Histopaque 1077; Sigma-Aldrich, Milan, Italy) and centrifuged at $950 \boldsymbol{g}$ for $20 \mathrm{~min}$. To separate monocytes from lymphocytes, $1 \mathrm{ml}$ of the cell suspension was added to a hyperosmotic Percoll solution (Sigma-Aldrich) in $3 \mathrm{ml}$ final volume and centrifuged at $580 \boldsymbol{g}$ for $15 \mathrm{~min}$. Collected cells were added with Dulbecco's PBS up to $15 \mathrm{ml}$, centrifuged at $350 \boldsymbol{g}$ for $7 \mathrm{~min}$, thus obtaining a monocyte-enriched suspension. To discard platelets and dead cells, in each centrifuge tube, $3 \mathrm{ml}$ of the iso-osmotic Percoll solution was overlaid with $1 \mathrm{ml}$ of the monocyte-enriched suspension and centrifuged at $350 \boldsymbol{g}$ for $15 \mathrm{~min}$; newly resuspended in Dulbecco's-PBS and finally centrifuged at $350 \mathrm{~g}$ for $7 \mathrm{~min}$, storing the pellet at $-80^{\circ} \mathrm{C}$.

\section{Cytokine gene expression}

IL-1 $\beta$, IL- 6 and IL- 8 mRNA levels were evaluated by RT-PCR from total RNA extracts obtained by the RNeasy mini kit (Qiagen, Milano, Italy). Total RNA was reverse-transcribed using the ImProm II ${ }^{\mathrm{TM}}$ RT-PCR System (Promega, Madison, WI, USA). Amplification primers for IL-1 $\beta$, IL- 6 and IL- 8 and the housekeeping gene glyceraldehyde 3-phosphate dehydrogenase were as follows: IL- $1 \beta$ sense $5^{\prime}$-GGATATGGAGCAACAAGTGG-3', antisense 5'-ATGTACCAGTTGGGGAACTG-3'; IL-6 sense 5'-GTCTCCTCATTGAATCCAGATTGG-3', antisense 5'-AGCTCAGCTATGAACTCCTTCTC-3'; IL-8 sense 5'-TCTTGCACAAATATTTGATGC- $3^{\prime}$, antisense $5^{\prime}$-CCACTGTGCCTTGGTTTC-3'; glyceraldehyde 3-phosphate dehydrogenase sense 5'-GTGAGGAGGGGAGATTCA-3', antisense 5'-GCATCCTGGGCTACACTG- $3^{\prime}$. Oligonucleotides were synthesised by M-Medical Genenco-Life Science (Firenze, Italy). Amplification was carried out with a Platinum PCR SuperMix High Fidelity kit (Invitrogen, Milano, Italy). Products were separated on $1 \%$ agarose, stained with ethidium bromide and quantified by scanning densitometry.

To more precisely compare gene expression between the two groups, real-time PCR analysis was also performed. For first-strand complementary DNA synthesis, $1 \mu \mathrm{g}$ RNA was reverse-transcribed in a $20 \mu \mathrm{l}$ volume using random hexamers as primers, according to the manufacturer's instructions (First Strand complementary DNA Synthesis Kit for RT-PCR; AMV, Roche, Indianapolis, IN, USA). The primers and probe sequences were obtained from PE Applied Biosystems (PreDeveloped TaqMan Assay Reagents Control Kits; Foster City, CA, USA). The following assay ID were used: IL-1 $\beta$, Hs00174097m1; IL-6, Hs00174131m1; IL-8, Hs00174103m1; glyceraldehyde 3-phosphate dehydrogenase, Hs99999905m1. PCR amplifications were performed in a total volume of $20 \mu \mathrm{l}$ containing $20 \mathrm{ng}$ of the complementary DNA sample, $200 \mathrm{nmol} / \mathrm{l}$ of each primer, $100 \mathrm{nmol} / 1$ of the corresponding probe, $12.5 \mu \mathrm{l}$ of the TaqMan Universal PCR Master Mix. For each reaction, the polymerase was activated by preincubation at $95^{\circ} \mathrm{C}$ for $10 \mathrm{~min}$. Amplification was then performed by forty cycles of switching between $95^{\circ} \mathrm{C}$ for $15 \mathrm{~s}$ and $60^{\circ} \mathrm{C}$ for $60 \mathrm{~s}$ The quantity of each complementary DNA sample was normalised to the housekeeping gene glyceraldehyde 3-phosphate dehydrogenase. 


\section{Release of markers of inflammation and oxidative stress}

IL-6, sCD40L, soluble VCAM-1, soluble ICAM-1 and plasma nitrotyrosine concentrations were analysed using commercially available ELISA (R\&D Systems, Abingdon, UK and Cell Sciences, Canton, MA, USA).

\section{Statistical analysis}

Given the exploratory nature of the study, no prespecified outcome variable was selected, and a formal power analysis was not carried out. Plasma insulin and TAG levels were non-normally distributed and were therefore log-transformed for use in statistical analyses. Comparisons of baseline data were performed by the unpaired Student's $t$ test. Differential changes between the two groups over time were tested by two-way ANOVA for repeated measures; results are given as significance of the time factor and the group $\times$ time interaction term. Simple and multiple regression analyses were performed by standard methods. Data were analysed using the StatView software package (SAS Institute Inc., Cary, NC, USA).

\section{Results}

FHD+ subjects were slightly older and heavier than FHDsubjects and showed a trend towards higher blood pressure and cholesterol levels; however, a slightly higher plasma TAG concentration was the only significantly different variable between the two groups (data not shown).

Following the test meal, TAG, NEFA and insulin all rose significantly, while total cholesterol and plasma glucose concentrations remained stable (Table 1). These changes were similar in the two groups.

No significant difference in the monocyte expression of the three cytokines was found between the FHD+ and FHDsubjects, either basally or at $6 \mathrm{~h}$ post-meal, nor did the meal alter the baseline expression pattern. This observation was confirmed by the values obtained by real-time PCR: no difference in the expressions of IL- 6 , IL- 8 and IL- $1 \beta$ before and after the meal was observed either in FHD+ or FHD - subjects (Fig. 1). Moreover, median plasma IL-6 levels, similar in the two groups in the fasting state ( 0.43 (interquartile range $0 \cdot 27) \mathrm{pg} / \mathrm{ml}$ in $\mathrm{FHD}-$ subjects and 0.57 (interquartile range $0.47) \mathrm{pg} / \mathrm{ml}$ in FHD+ subjects), did not change significantly at 2,6 or $12 \mathrm{~h}(0.56$ (interquartile range 0.33$) v .0 .53$ (interquartile range 0.49 ); 0.70 (interquartile range 0.55$) v .0 .63$ (interquartile range 0.55$) ; 0.54$ (interquartile range 0.45$) v .0 .58$ (interquartile range 0.58$) \mathrm{pg} / \mathrm{ml}$, all $P=\mathrm{NS}$ for either a group or group $\times$ time interaction). Median sCD40L levels followed the same trend, without any differences between the two groups either in the fasting state (2.48 (interquartile range $1.77) \mathrm{ng} / \mathrm{ml}$ in $\mathrm{FHD}-$ subjects and 1.69 (interquartile range $4 \cdot 28) \mathrm{ng} / \mathrm{ml}$ in FHD+ subjects) or in the post-meal state (2.06 (interquartile range 1.71) v. 1.68 (interquartile range 3.92 ) and 2.07 (interquartile range 1.83) v. 1.27 (interquartile range 3.12 ) at 2 and $6 \mathrm{~h}$, respectively, both $P=\mathrm{NS}$ ). After $12 \mathrm{~h}$, median sCD40L concentration was $2 \cdot 15$ (interquartile range 1.21$) \mathrm{ng} / \mathrm{ml}$ in $\mathrm{FHD}-$ subjects and 1.13 (interquartile range 2.93$)) \mathrm{ng} / \mathrm{ml}$ in $\mathrm{FHD}+$ subjects, $P=\mathrm{NS}$ for either a group or group $\times$ time interaction.

With regard to the serum levels of the subjects' adhesion molecules, neither VCAM-1 nor ICAM-1 changed in FHDsubjects, while both increased in FHD+ subjects significantly, though still within the normal range $(P \leq 0.01$ for the group $\times$ time interaction; Fig. 2). For both markers, the between-group difference persisted after adjusting (by multivariate regression) for age, insulin, total cholesterol or TAG. At $12 \mathrm{~h}$, VCAM-1 and ICAM-1 tended to decline but were still significantly higher in FHD + subjects. Nitrotyrosine levels were superimposable between the FHD - and FHD+ subjects at baseline but rose significantly higher in FHD+ subjects than in FHDsubjects at 2 and $6 \mathrm{~h}$ post-meal (Fig. 2); after $12 \mathrm{~h}$, this difference was cancelled. In FHD+ subjects, nitrotyrosine was directly related to NEFA $(r 0.53, P<0.0001)$, TAG $(r 0.32$, $P=0.03)$ and insulin $(r 0.36, P=0 \cdot 01)$ levels.

In the whole study group, there was a positive correlation between the meal-induced $\triangle$ VCAM-1 and the baseline $(r 0 \cdot 450, P=0 \cdot 0098)$ and post-meal $(r 0.514, P=0 \cdot 0031)$ TAG levels, while meal-induced $\triangle \mathrm{ICAM}-1$ was significantly related to meal-induced nitrotyrosine $(r 0.537, P=0.0015)$.

Table 1. Biochemical parameters before and after a fat-enriched breakfast in subjects with (FHD+ ) or without (FHD- ) a family history of type 2 diabetes

(Mean values and standard deviations)

\begin{tabular}{|c|c|c|c|c|c|c|c|c|c|}
\hline & \multirow[b]{2}{*}{ Group } & \multicolumn{2}{|c|}{ Pre } & \multicolumn{2}{|c|}{$2 \mathrm{~h}$} & \multicolumn{2}{|c|}{$6 \mathrm{~h}$} & \multicolumn{2}{|c|}{$P^{*}$} \\
\hline & & Mean & SD & Mean & SD & Mean & SD & $G$ & $T$ \\
\hline \multirow[t]{2}{*}{ Glucose (mmol/l) } & FHD- & 4.7 & 0.4 & 4.8 & 0.2 & 4.9 & 0.3 & NS & NS \\
\hline & FHD+ & $4 \cdot 8$ & 0.4 & 4.9 & 0.2 & 4.9 & 0.3 & & \\
\hline \multirow[t]{2}{*}{ Cholesterol $(\mathrm{mmol} / \mathrm{l})$} & FHD- & 4.90 & 1.01 & 4.95 & 0.96 & $4 \cdot 87$ & $0 \cdot 88$ & NS & NS \\
\hline & $\mathrm{FHD}+$ & $5 \cdot 34$ & 1.04 & $5 \cdot 39$ & 1.01 & $5 \cdot 31$ & 0.98 & & \\
\hline \multirow[t]{2}{*}{ Insulin (pmol/l) } & FHD- & 65 & 25 & 109 & 27 & 54 & 24 & NS & $<0.0001$ \\
\hline & FHD+ & 74 & 49 & 119 & 38 & 62 & 28 & & \\
\hline \multirow[t]{2}{*}{ TAG $(\mathrm{mmol} / \mathrm{l})$} & FHD- & 0.74 & 0.19 & 0.90 & 0.20 & 0.84 & 0.21 & 0.02 & $<0.0001$ \\
\hline & FHD+ & 0.96 & 0.24 & $1 \cdot 13$ & 0.35 & $1 \cdot 22$ & 0.40 & & \\
\hline \multirow[t]{2}{*}{ NEFA $(\mu \mathrm{mol} / \mathrm{l})$} & FHD- & 401 & 231 & 485 & 171 & 614 & 196 & NS & $<0.0001$ \\
\hline & $\mathrm{FHD}+$ & 493 & 254 & 594 & 264 & 744 & 296 & & \\
\hline
\end{tabular}

$T$, time; $\mathrm{G}$, group $\times$ time interaction.

${ }^{*} P$ value for the effect of $T$ and $G$. 

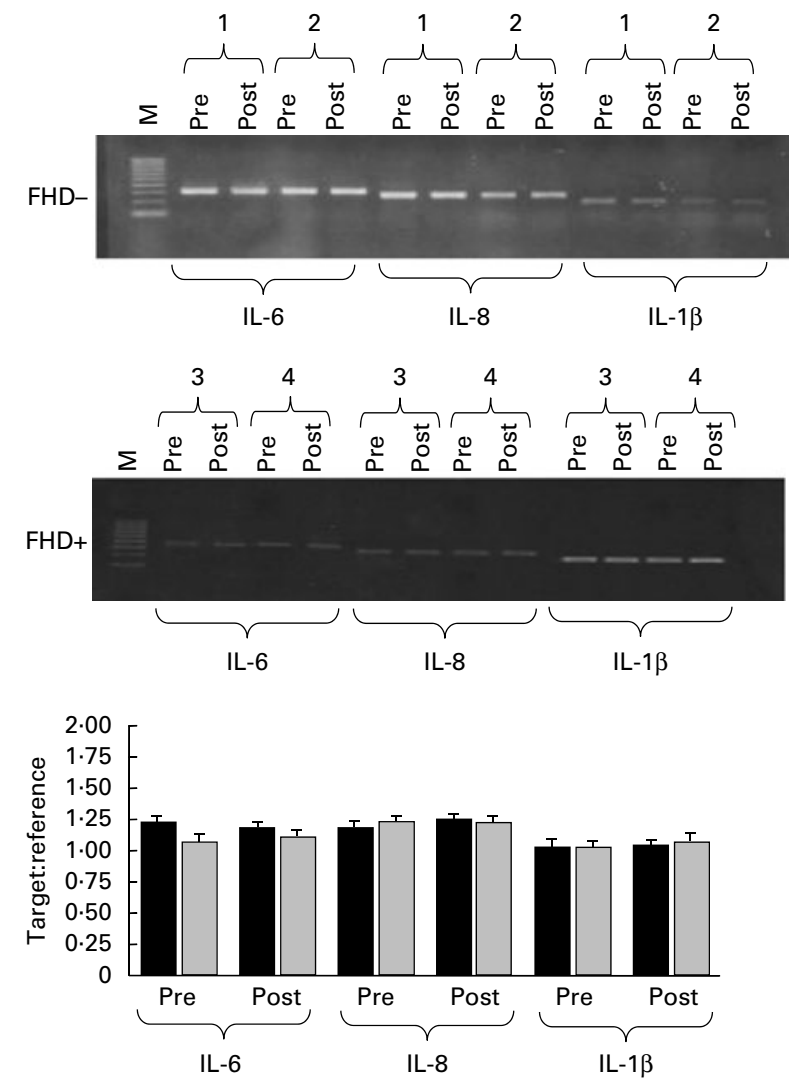

Fig. 1. Representative RT-PCR for the monocyte mRNA expressions of IL-6, IL- 8 and IL-1 $\beta$ in two subjects without a family history of type 2 diabetes (FHD-; no. 1 and 2, representative of sixteen) and two subjects with a family history of type 2 diabetes (FHD+; no. 3 and 4, representative of sixteen) in the fasting state (pre) and $6 \mathrm{~h}$ after a fat-enriched breakfast (post), and the quantitative evaluation of IL- $6, \mathrm{IL}-8$ and IL-1 $\beta$ expressions in sixteen FHD - and sixteen FHD+ subjects measured by real-time PCR in the fasting state (pre) and $6 \mathrm{~h}$ after a fat-enriched breakfast (post). Values are means, with standard errors represented by vertical bars. $\mathbf{q}, \mathrm{FHD}-; \square$, FHD+; M, marker.

\section{Discussion}

In the present study, we show that young healthy subjects with a parental history of T2D have a normal response to an oral fat load in terms of inflammatory cytokines expression and release; in contrast, the response of the circulating adhesion molecules is modestly but consistently increased in parallel with a meal-induced rise in nitrotyrosine, an index of oxidative stress. These data do not confirm those by Nappo et $a l .{ }^{(16)}$, who found an enhanced IL-6 and TNF- $\alpha$ release after either a glucose or a fat load also in normal subjects. One explanation for this discrepancy is that our subjects were carefully selected for being free from confounding factors such as glucose intolerance, obesity or hypertension. The test meal that we used did not induce changes in either IL-1 $\beta$, a cytokine mainly released by neutrophils that might play a role in inducing $\beta$-cell apoptosis ${ }^{(17)}$, or IL-6, a marker of insulin resistance whose levels have been shown to predict the risk of incident diabetes ${ }^{(18,19)}$, unmodified by a fatty meal in normal individuals as reported by Poppitt et al. ${ }^{(20)}$. This suggests that family history of diabetes per se, in the absence of overt metabolic abnormalities, does not confer susceptibility to an inflammatory response to an acute stimulus represented by a lipid oral load. Similarly, the pro-inflammatory mediator sCD40L, which is expressed in CD4+T cells and activated platelets and participates in several inflammatory and pro-atherothrombotic functions ${ }^{(21)}$, was unaffected by an oral fat load in both groups, implying that familial predisposition to diabetes does not add prothrombotic risk.

At variance with the observations made by Ceriello et al. ${ }^{(22)}$, VCAM-1 and ICAM-1 did not increase post-meal in FHDsubjects, but did so in FHD+ subjects, even remaining within the normal range, suggesting an early defect in vascular reactivity and endothelial function in these subjects, possibly outlined by the oral fat load. In this respect, the direct relationship between TAG and VCAM-1 appears of particular interest, supporting the role of circulating lipids in endothelial function $^{(23)}$. We actually show that not only post-meal TAG but even fasting TAG might play a role in determining VCAM-1 variation in the post-absorptive phase, reinforcing the role of
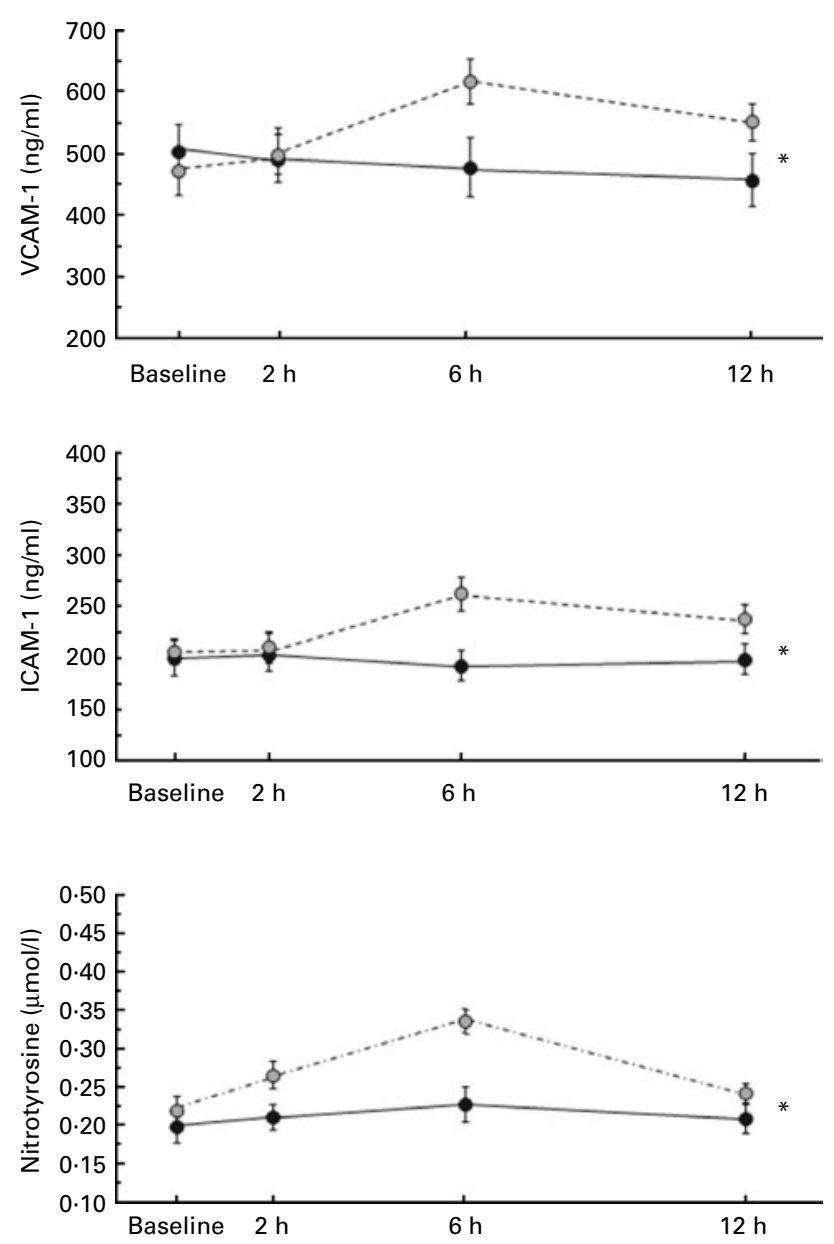

Fig. 2. Circulating levels of vascular cellular adhesion molecule-1 (VCAM-1) intracellular adhesion molecule-1 (ICAM-1) and nitrotyrosine at baseline and 2,6 and $12 \mathrm{~h}$ after a fat-enriched breakfast in subjects with $(\mathrm{FHD}+)$ and without (FHD-) a family history of type 2 diabetes. Values are means, with standard errors represented by vertical bars. * Mean values were significantly different for the group $\times$ time interaction along the whole test $(P \leq 0.01)$. FHD-; O, FHD+. 
lipid metabolism as a strong determinant of endothelial function. The rise in nitrotyrosine levels (a useful marker of both peroxynitrite and nitric oxide activity) in the FHD+ subjects and their relation to TAG and FFA levels resonate with the concept that NEFA increase oxidative stress generation in humans ${ }^{(24)}$. Further support to such a hypothesis might come from the relationship between the variation of ICAM-1 and nitrotyrosine levels, while such a correlation with VCAM-1 is lacking. This may be referred to the differences between these two adhesion molecules, given that ICAM-1 is mostly expressed at the level of endothelial cells, and its expression is enhanced by a variety of pro-inflammatory stimuli ${ }^{(25)}$.

In conclusion, in young healthy subjects, parental diabetes appears to be associated with the evidence of abnormal endothelial reactivity and increased oxidative stress in response to an acute stimulus. The present study has several limitations, mainly its exploratory, hypothesis-generating nature and the small sample size. These observations await to be confirmed in larger cohorts of subjects.

\section{Acknowledgements}

The present study received no specific grant from any funding agency in the public, commercial or not-for-profit sectors. S. M. did the majority of laboratory determinations. V. C. recruited the volunteers and performed the tests. E. S. gave an important contribution to the experimental procedures. E. F. is the head of the section; he revised the manuscript and gave important suggestions to improve the quality of the manuscript. A. S. designed the experimental protocol, analysed the data and wrote the manuscript. The authors have no conflict of interest to disclose.

\section{References}

1. Nyholm B, Nielsen MF, Kristensen K, et al. (2004) Evidence of increased visceral obesity and reduced physical fitness in healthy insulin-resistant first-degree relatives of type 2 diabetic patients. Eur J Endocrinol 150, 207-214.

2. Cnop M, Vidal J, Hull RL, et al. (2007) Progressive loss of beta-cell function leads to worsening glucose tolerance in first-degree relatives of subjects with type 2 diabetes. Diabetes Care 30, 677-682.

3. Eberly LE, Stamler J \& Neaton JD (2003) Relation of triglyceride levels, fasting and nonfasting, to fatal and nonfatal coronary heart disease. Arch Intern Med 163, 1077-1083.

4. Boquist S, Ruotolo G, Tang R, et al. (1999) Alimentary lipemia, postprandial triglyceride-rich lipoproteins, and common carotid intima-media thickness in healthy, middleaged men. Circulation 100, 723-728.

5. Damjanovic M \& Barton M (2008) Fat intake and cardiovascular response. Curr Hypertens Rep 10, 25-31.

6. Keogh JB, Grieger JA, Noakes M, et al. (2005) Flow-mediated dilatation is impaired by a high-saturated fat diet but not by a high-carbohydrate diet. Arterioscler Thromb Vasc Biol 25, 1274-1279.

7. Aronson D (2008) Hyperglycemia and the pathobiology of diabetic complications. Adv Cardiol 45, 1-16.
8. Fantuzzi G (2005) Adipose tissue, adipokines, and inflammation. J Allergy Clin Immunol 115, 911-919.

9. Henrichot E, Juge-Aubry CE, Pernin A, et al. (2005) Production of chemokines by perivascular adipose tissue: a role in the pathogenesis of atherosclerosis? Arterioscler Thromb Vasc Biol 25, 2594-2599.

10. Li G, Sanders JM, Bevard MH, et al. (2008) CD40 ligand promotes Mac-1 expression, leukocyte recruitment, and neointima formation after vascular injury. Am J Pathol 172, $1141-1152$.

11. Varo N, Libby P, Nuzzo R, et al. (2005) Elevated release of sCD40L from platelets of diabetic patients by thrombin, glucose and advanced glycation end products. Diab VasC Dis Res 2, 81-87.

12. Patel JV, Lim HS, Nadar S, et al. (2006) Abnormal soluble CD40 ligand and C-reactive protein concentrations in hypertension: relationship to indices of angiogenesis. J Hypertens 24, 117-121.

13. Chakrabarti S, Blair P \& Freedman JE (2007) CD40-40L signaling in vascular inflammation. $J$ Biol Chem 282, 18307-18317

14. Buolbou MS, Koukoulis GN, Makri ED, et al. (2005) Circulating adhesion molecules levels in type 2 diabetes mellitus and hypertension. Int J Cardiol 98, 39-44.

15. Meigs JB, Hu FB, Rifai N, et al. (2004) Biomarkers of endothelial dysfunction and risk of type 2 diabetes mellitus. JAMA 291, 1978-1986.

16. Nappo F, Esposito K, Cioffi M, et al. (2002) Postprandial endothelial activation in healthy subjects and in type 2 diabetic patients: role of fat and carbohydrate meals. $J \mathrm{Am}$ Coll Cardiol 39, 1145-1150.

17. Donath MY, Schumann DM, Faulenbach M, et al. (2008) Islet inflammation in type 2 diabetes: from metabolic stress to therapy. Diabetes Care 31, S161-S164.

18. Hu FB, Meigs JB, Li TY, et al. (2004) Inflammatory markers and risk of developing type 2 diabetes in women. Diabetes 53, 693-700.

19. Pradhan AD, Manson JE, Rifai N, et al. (2001) C-Reactive protein, interleukin 6 , and risk of developing type 2 diabetes mellitus. JAMA 286, 327-334.

20. Poppitt SD, Keogh GF, Lithander FE, et al. (2008) Postprandial response of adiponectin, interleukin-6, tumor necrosis factor- $\alpha$, and C-reactive protein to a high-fat dietary load. Nutrition 24, 322-329.

21. Schonbeck U \& Libby P (2001) CD40 signaling and plaque instability. Circ Res 89, 1092-1103.

22. Ceriello A, Quagliaro L, Piconi L, et al. (2004) Effect of postprandial hypertriglyceridemia and hyperglycemia on circulating adhesion molecules and oxidative stress generation and the possible role of simvastatin treatment. Diabetes $\mathbf{5 3}$, $701-710$.

23. Rubin D, Claas S, Pfeuffer M, et al. (2008) s-ICAM-1 and sVCAM-1 in healthy men are strongly associated with traits of the metabolic syndrome, becoming evident in the postprandial response to a lipid-rich meal. Lipids Health Dis $\mathbf{1}$, $7-32$.

24. Steinberg HO, Tarshoby M, Monestel R, et al. (1997) Elevated circulating free fatty acid levels impair endothelium-dependent vasodilation. J Clin Invest 100, 1230-1239.

25. Wallen $\mathrm{NH}$, Held C, Rehnqvist N, et al. (1999) Elevated serum intercellular adhesion molecule-1 and vascular adhesion molecule- 1 among patients with stable angina pectoris who suffer cardiovascular death or non-fatal myocardial infarction. Eur Heart J 20, 1039-1043. 